\title{
REVISÃO INTEGRATIVA ACERCA DA PERCEPÇÃO DA MULHER QUE NÃO CONSEGUE EXERCER O PAPEL DA MATERNIDADE
}

\author{
INTEGRATIVE REVIEW ABOUT THE PERCEPTION OF WOMEN WHO CAN \\ NOT EXERCISE THE ROLE OF MATERNITY
}

\author{
Emanuella Alexandre Estrela, Juliana Leilany de Lima Dantas, Ellany Gurgel Cosme do \\ Nascimento
}

Universidade do Estado do Rio Grande do Norte - UERN

\begin{abstract}
The objective of this study is to capture in scientific publications the perception of women who can not play the role of motherhood. This is an integrative review. The search for the articles was carried out in the databases: Scientific Eletronic Library Online (SciELO) and Public Medline or Publisher Medline (PUBMED). For the discussion of this research, the criterion of analysis of the topics that provided to categorize, elucidate and classify the data was applied. This grouping shows two categories: The impact of infertility on the life of the couple and the idealization of the biological child. It is still perceptible how the woman is seen relative to the society when speaking of being a mother. For they see the woman responsible only for bearing a child, for being a mother. Society imposes on these women who present difficulties such as being infertile, showing feelings of inferiority and negativity, this is very present in the presentations of the articles used. The importance of the participation of health professionals is fundamental for an approach and reception, capable of having a close look to perceive women seeking the service by the need for infertility, for this they need to be sensitive, paying attention to humanized Decent work for these women.
\end{abstract}

Keywords: Female Infertility; Conjugal State; Women's health.

\section{Resumo}

O Objetivo deste estudo é averiguar nas publicações científicas os sentimentos expericiados pela mulher que não consegue exercer o papel da maternidade. Trata-se de uma revisão integrativa. A busca dos artigos foi realizada nas bases de dados: $A$ Scientific Eletronic Library Online (SciELO) e na Public Medline or Publisher Medline (PUBMED). Para a discussão desta pesquisa, foi aplicado o critério de análise dos tópicos que proporcionou categorizar, elucidar e classificar os dados. Desse agrupamento apresentam-se duas categorias: $O$ impacto da infertilidade na vida do casal e a idealização do filho biológico. Ainda é perceptível como a mulher é vista parente a sociedade ao falar-se de ser mãe. Onde na maioria das vezes, essa mulher é vista apenas como responsável em gerar um filho. A sociedade impõe muito desse grupo de mulheres, e por consequência disso elas demonstram sentimentos de inferioridade $e$ negatividade. A importância da participação dos profissionais de saúde é fundamental para uma abordagem e acolhimento eficaz, podendo ser capazes de ter um olhar minucioso, percebendo assim as mulheres que procuram o serviço pela necessidade referente a infertilidade, para isso eles precisam ser sensíveis, prestando uma atenção humanizada e assim conseguiram promover uma rede de assistência digna para essas mulheres.

Palavras-chaves: Infertilidade Feminina; Estado Conjugal; Saúde da mulher. 
Introdução

Segundo a Organização Mundial da Saúde (OMS), infertilidade é a incapacidade de um casal gerar um filho no decorrer de um ano sem o uso de métodos contraceptivos ${ }^{1}$.

Estima-se que cerca de $8 \%$ a $15 \%$ dos casais em idade reprodutiva no mundo, que se deparam com dificuldade para a concepção de um filho ${ }^{2}$. No Brasil, supõe que existam 51,2 milhões de mulheres em idade reprodutiva, o que proporcionalmente indica que existem de 4 milhões a 7,68 milhões de mulheres inférteis ${ }^{3}$.

No Brasil, mais de 278 mil casais têm dificuldade de gerar um filho em algum momento da vida, devido a alguma forma de infertilidade, primária ou secundária. A infertilidade primária é aquela em que o casal nunca concebeu e a secundária ocorre quando o casal possui um ou mais concepto ${ }^{4}$.

A infertilidade gera muitas limitações na vida dos casais impedindo que os mesmos possam da continuidade a vida através de um filho biológico. A infertilidade é considerada um problema do casal, porém a mulher é vista como a principal responsável pela problemática da infertilidade ${ }^{5}$.

As mulheres são socialmente convictas de que ser mãe é essencial para se sentirem completas e realizadas, cabendo a elas o comprometimento pelo sucesso dessa função. 0 fato de que assuntos associados à criança e a fecundação fazerem parte da população feminina, quando acontece a infertilidade, normalmente, a maior responsabilidade recai sobre as mulheres. Consequentemente em meio à naturalidade do valor social da maternidade, por tantas vezes essas mulheres se sentem culpadas e inferiores devido à incapacidade de cumprir a função parental tão esperada e cobrada pela sociedade. Diante dessa imposição social da maternidade para a "mulher com capacidade reprodutiva, a mulher infértil se sente menos feminina e, consequentemente, menos atraente". Sendo que os estudos raramente fazem referência à infertilidade masculina, tornando assim, um peso maior para a vida da mulher 6 .

A mulher quando diagnosticada como infértil encontra-se com sensações de tristeza, de incompletude, solidão e inferioridade. Ao perceber que a efetuação do seu desejo não está em suas mãos, sentem-se impotentes, fracassadas, humilhadas, desamparadas e injustiçadas, manifestando vestígios de depressão, inquietude e desânimo ${ }^{7}$. Apto a ocasionar uma queda na auto-estima feminina, a infertilidade é, muitas vezes, sentida como defeito, ocasionando um sentimento de desprestígio e afetando também outras esferas da vida ${ }^{6}$

A realização do presente trabalho tem como objetivo averiguar nas publicações científicas os sentimentos vivenciados pela mulher que não consegue exercer o papel da maternidade.

\section{Metodologia}

Trata-se de um estudo de revisão integrativa de artigos científicos que abordaram a infertilidade feminina. A busca dos artigos foi realizada em setembro de 2016 nas bases de dados: A Scientific Eletronic Library Online (SciELO) e na Public Medline or Publisher Medline (PUBMED). Sendo utilizado o termo "Infertility And Maternity" para a pesquisa em ambas as bases, estabelecendo os seguintes critérios de inclusão: artigos de livre acesso, em língua portuguesa e em período livre. No critério de exclusão: sendo artigos não disponíveis na íntegra; artigos de revisão, dissertação e tese.

A princípio, a busca foi realizada na base de dados SciElo, obtendo um total de 14 artigos, após a aplicação do filtro português, resultou 13 artigos. Em seguida foi realizado a busca na Pubmed, resultando 560 artigos, após utilizado do filtro livre o resultado foi de 138 artigos, e com idioma português 03 artigos. A pesquisa teve um total 16 artigos científicos, sendo excluídos as duplicidades e títulos, resultando um total de 14 artigos.

Com base no título dos artigos, foi realizada a exclusão dos artigos que não tinham afinidade com o objetivo do presente estudo e os artigos duplicados. Sendo eliminados pelo 10 Avaliador 06 artigos a partir da leitura dos resumos, e pelo 20 Avaliador 05 artigos, após a uma breve conversa entre os dois avaliadores foram excluídos 05 artigos. Resultando no total 09 artigos.

Foi executada uma analise a partir das leituras dos resumos, sucedendo a exclusão de um artigo teórico conceitual. Em vista disso, foi consolidado um total de 07 artigos referidos as bases de dados.

\section{Resultados e Discussões}

Os trabalhos científicos analisados possibilitaram verificar como está procedendo o crescimento do conhecimento da infertilidade e a 
relação do papel da maternidade da mulher no Brasil, no período de 2000 a 2014. Esses estudos colaboraram para a certificação de como essas pessoas vivem sobre a condição da infertilidade.

Foram compiladas as informações dos artigos, para facilitar a análise, a partir da organização e exposição dos resultados, destacando-se: os autores da pesquisa; ano de publicação; objetivo da pesquisa; tipo de estudo; tamanho amostral e revista de publicação (Tabela 1)

Tabela 1 - Caracterização das publicações científicas no Brasil, relacionadas à Infertilidade And Maternidade, no período de 2000 a 2014. Pau dos Ferros/2016.

\begin{tabular}{|c|c|c|c|c|c|}
\hline Autores & $\begin{array}{c}\text { Ano de } \\
\text { publicação }\end{array}$ & Objetivo & $\begin{array}{l}\text { Tipo de } \\
\text { Estudo }\end{array}$ & $\begin{array}{l}\text { Tamanho } \\
\text { amostral }\end{array}$ & $\begin{array}{l}\text { Revista de } \\
\text { Publicação }\end{array}$ \\
\hline $\begin{array}{c}\text { LINS et } \\
\text { al }\end{array}$ & 2014 & $\begin{array}{l}\text { Investigar, nas mulheres entrevistadas, } \\
\text { os aspectos inconscientes do desejo } \\
\text { singular pela maternidade e a dificuldade } \\
\text { do feminino em lidar com a } \\
\text { impossibilidade de gerar um filho. }\end{array}$ & Qualitativo & $\begin{array}{c}200 \\
\text { sujeitos }\end{array}$ & $\begin{array}{l}\text { Estudos de } \\
\text { psicologia } \\
\text { (Campinas) }\end{array}$ \\
\hline $\begin{array}{c}\text { LEITE et } \\
\text { al }\end{array}$ & 2014 & $\begin{array}{l}\text { Compreender como a infertilidade afeta } \\
\text { a vida e a autoimagem feminina de } \\
\text { mulheres que estão passando, ou que já } \\
\text { passaram, por um tratamento atribuem à } \\
\text { maternidade e ao"ser mulher". }\end{array}$ & Qualitativo & 4 sujeitos & $\begin{array}{c}\text { Abordagem } \\
\text { Gestáltica }\end{array}$ \\
\hline $\begin{array}{l}\text { LANIUS } \\
\text { et al }\end{array}$ & 2010 & $\begin{array}{c}\text { Este estudo apontará alguns impasses do } \\
\text { desejo expressos pela mulher infértil que } \\
\text { busca, nas chamadas } \\
\text { Novas Tecnologias de Reprodução } \\
\text { Humana Assistida, um recurso para } \\
\text { poder engravidar e ter seu filho } \\
\text { consangüíneo }\end{array}$ & Relato & 4 sujeitos & $\begin{array}{l}\text { Latinoameri } \\
\text { cana de } \\
\text { Psicopatolo } \\
\quad \text { gia } \\
\text { Fundament } \\
\quad \text { al }\end{array}$ \\
\hline $\begin{array}{c}\text { MATOS } \\
\text { et al }\end{array}$ & 2000 & $\begin{array}{l}\text { Pretende-se analisar os conceitos de } \\
\text { feminino e de materno considerando-os } \\
\text { como factores psicológicos que poderão } \\
\text { interferir ou influenciar o desejo e/ou a } \\
\text { capacidade de ter filhos. }\end{array}$ & Qualitativo & $\begin{array}{c}22 \\
\text { sujeitos }\end{array}$ & $\begin{array}{l}\text { Psicologia, } \\
\text { Saúde \& } \\
\text { Doenças }\end{array}$ \\
\hline $\begin{array}{l}\text { BORLOT } \\
\text { et al }\end{array}$ & 2004 & $\begin{array}{l}\text { O objetivo dessa pesquisa foi investigar: } \\
\text { a trajetória percorrida pelos casais que, } \\
\text { tentando resolver o problema da } \\
\text { infertilidade, submeteram-se à } \\
\text { inseminação artificial, assim como o } \\
\text { significado da presença de um filho } \\
\text { biológico para esses casais }\end{array}$ & Qualitativo & $\begin{array}{c}10 \\
\text { sujeitos }\end{array}$ & $\begin{array}{l}\text { Estudos de } \\
\text { psicologia }\end{array}$ \\
\hline $\begin{array}{l}\text { DORNEL } \\
\text { LES et al }\end{array}$ & 2011 & $\begin{array}{l}\text { Conhecer o processo de tornar-se mãe } \\
\text { em decorrência de infertilidade no casal, } \\
\text { é possível recorrer às técnicas de } \\
\text { reprodução assistida para a realização } \\
\text { desse projeto }\end{array}$ & Qualitativo & 3 sujeitos & $\begin{array}{l}\text { Estudos de } \\
\text { psicologia } \\
\text { (Campinas) }\end{array}$ \\
\hline $\begin{array}{l}\text { GRADV } \\
\text { OHL et } \\
\text { al }\end{array}$ & 2013 & $\begin{array}{l}\text { O objetivo deste trabalho foi avaliar o } \\
\text { estresse de homens e mulheres, } \\
\text { membros de casais inférteis, no mo- } \\
\text { mento em que fazem a primeira consulta } \\
\text { em um serviço especializado para } \\
\text { tratamento de infertilidade, e identificar } \\
\text { fatores associados à sua ocorrência }\end{array}$ & Transversal & $\begin{array}{c}202 \\
\text { sujeitos }\end{array}$ & $\begin{array}{l}\text { Revista } \\
\text { Brasileira de } \\
\text { Ginecologia } \\
\text { e } \\
\text { Obstetrícia }\end{array}$ \\
\hline
\end{tabular}

Assim, para a discussão desta pesquisa, foi aplicado o critério de análise dos tópicos que proporcionou categorizar, elucidar e classificar os dados. Desse agrupamento mostram-se duas categorias: 1) O impacto da infertilidade na vida do casal, apontando 3 artigos. 2) A idealização do filho biológico, apresentando 4 artigos. Em seguida percorre os principais achados dos artigos referente à Tabela 1. 
No primeiro artigo, o sentido da maternidade e da infertilidade: um discurso singular aborda o desejo da mulher de ser mãe associado à busca de uma identificação feminina do ser mulher pela via da maternidade. E a sua impossibilidade de gerar um filho.

No segundo artigo, o desejo de ser mãe e a barreira da infertilidade: uma compreensão fenomenológica que aborda como a infertilidade afeta a vida e auto-imagem das mulheres que buscaram ou estão em um tratamento para a realização de ser mãe.

No terceiro artigo, reprodução assistida: os impasses do desejo vêm abordando a importância do filho consanguíneo para a mulher, levando-a a procurar as clínicas de reprodução assistida para essa realização.

No quarto artigo, validação preliminar de uma técnica de avaliação de feminino/materno, aborda uma análise dos conceitos de feminino e materno considerando os fatores psicológicos poderão interferir e influenciar no desejo e na capacidade dessas mulheres gerarem filhos.

No quinto artigo, as tecnologias de reprodução assistida e as representações sociais de filho biológico abordam a busca pela a realização de um filho biológico para os casais, que optaram por inseminação artificial para a realização de se tornarem pais biológicos.

No sexto artigo, será que eu consigo levar essa gestação até o fim? A experiência materna da gestação no contexto da reprodução assistida aborda o conhecimento do processo de tornar-se mãe, através da técnica de reprodução assistida, mostrando os sentimentos de várias mulheres ao realizar essa técnica, sendo eles o medo de não engravidar e de levar a gestação até o fim.

No sétimo artigo, estresse de homens e mulheres que buscam tratamento para infertilidade, aborda o estresse que é causado pela a busca de serviço especializado para tratamento de infertilidade e a identificação das causas relacionadas a sua situação.

\section{Discussão}

\section{Análise das categorias:}

\section{O impacto da infertilidade na vida do casal}

A infertilidade quando diagnosticada provoca uma instabilidade na vida dos casais, isso acontece, pois os dois almejam se tornarem pais, e depara-se com a dificuldade, com isso surgem consequências como: depressão, o medo do rompimento do relacionamento, impotência, tristeza, culpa, sensação de vazio, fragilidade e inferioridade. Por outro lado, muitos casais inférteis acabam se aproximando perante a experiência da infertilidade se tornando mais unidos e cúmplices ${ }^{10}$.

Os trabalhos sobre os aspectos psicológicos da infertilidade apresentam-se em dois aspectos, onde o primeiro refere-se a complexidade e dificuldades emocionais envolvidos na base da infertilidade, e a outra diz respeito às consequências que a infertilidade proporciona na vida do indivíduo e, conseguinte, na vida do casal $^{10}$.

Mediante o forte desejo da maternidade e paternidade, os casais inférteis também entram em contato com questões relacionadas a respeito da masculinidade e feminilidade. Sentem-se ofendidos no seu valor de ser homem e de ser mulher quando mutilados na sua função reprodutiva. De modo que, uma vez que o desejo da maternidade e paternidade se liga profundamente com as vivências representativas de cada pessoa, o conhecimento emocional de cada um sobre a infertilidade possui um caráter extremamente especifico ${ }^{8}$. Efetivamente, a infertilidade causa uma crise na vida do casal, exigindo uma mudança na sua vivencia sejam elas pessoais e conjugais, tornando-se uma de suas implicações a depressão pela ausência do corpo saudável e o medo da perda do seu relacionamento. O transtorno da infertilidade pode começar durante o transcurso da investigação para o próprio tratamento, os dois são momentos de grande destruição emocional que afetam na sexualidade do casal ${ }^{10}$.

A infertilidade é um "problema" que provoca um abrimento da ferida narcísica, modificando assim as funcionalidades essenciais do sujeito, e produzindo variáveis sintomas que muitas vezes prejudica relacionamentos familiares, conjugais, sexuais, sociais e profissionais $^{10}$.

Perante os possíveis grupos sociais entre as questões emocionais e a infertilidade, o acompanhamento psicológico em sido aconselhada, tanto como elemento de proteção à saúde mental dos casais inférteis, tanto quanto como um método para possibilitar uma mais adequada resposta aos tratamentos ${ }^{10}$.

\section{A idealização do filho biológico}

O filho biológico é idealizado desde muito cedo pelos seus futuros pais, porém quando isso é impedido que aconteça eles procuram formas 
para que seu "sonho" se torne real, buscando assim, por tratamentos que possibilitem essa idealização. Para eles o filho biológico tem uma representação importante, a continuidade de sua descendência, a própria geração do bebê, a apresentação de semelhanças física e outros. Isso possibilita que eles idealizem cada vez mais o surgimento e chegada de um filho carnal ${ }^{5}$. A imagem de que à mulher é responsável pela concepção de uma vida existe presente na sociedade de uma maneira geral, porém a infertilidade apresenta-se, para a maioria delas, como um peso enorme, consequentemente gerando sensação de culpa e pensamentos negativos, já que sua função é tida socialmente determinada pela maternidade ${ }^{5}$.

Por meio da maternidade, a mulher passou a ocupar um lugar relevante na estrutura familiar, sendo considerada necessária para a sobrevivência da família. A maternidade e o cuidado com o gerenciamento do lar se configuraram, assim, como funções femininas valorizadas socialmente, permitindo que a mulher fosse reconhecida e ocupasse uma posição de evidente predominância dentro da sociedade. Historicamente, a maternidade foi estabelecida como o ideal máximo da mulher, mostrando um caminho para alcançar a plenitude e a realização da feminilidade ${ }^{6}$.

Diante disso percebe-se que nos últimos anos esse cenário vem mudando, onde a mulher está situada em diferentes ambientes, possibilitando sua autonomia, e a escolha e a de não ser mãe.

Com mais acesso à educação formal e à formação profissional, as mulheres vão, no perpassar do século $\mathrm{XX}$, preencher progressivamente o espaço público, ao mesmo tempo em que mantêm a responsabilidade na criação dos filhos. Nesta circunstancia, ser ou não ser mãe passou a ter uma dimensão reflexiva ${ }^{8}$, a ser uma decisão racional, motivada por princípios relacionados às condições subjetivas, econômicas e sociais das mulheres e, também, do casal ${ }^{9}$.

A chegada da modernidade e de suas conquistas tecnológicas, acima de tudo no campo da contracepção, e mais ultimamente da concepção, trouxe às mulheres uma maior alternativa na escolha da maternidade e abrindo um espaço para criação do impasse de ser ou não ser mãe ${ }^{9}$.

Os homens e mulheres constroem um olhar ideal sobre o filho biológico, isso porque as representações sociais da infertilidade são intervindas por crenças e simbologias em torno da significância do filho biológico para o casal e para a manutenção do próprio casamento ${ }^{5}$. A representação social de filho biológico tem significado fortemente para os casais como: sangue do meu sangue, sendo que a gestação é dita como uma das partes mais importantes; a descendência, dando continuidade à família; as semelhanças físicas que os filhos teriam com os pais; e a pressão social influenciando as decisões do casal ${ }^{5}$.

Nos últimos anos houve um aumento na procura por clinicas de reprodução assistida por casais inférteis, para muitos deles essas técnicas de reprodução assistida é a última chance para a realização do sonho do filho biológico. Essas clínicas são geralmente procuradas depois de um longo período de tentativas dos casais. Porém, esses casais muitas vezes se deparam com problemas de ordem ética e legal trazidos pelo setor da reprodução assistida, sendo assim, é essencial reflexões por parte da comunidade sobre essa questão ${ }^{5}$.

\section{Conclusões}

Dentre os achados ficou perceptível como a mulher é vista parente a sociedade ao falar-se de ser mãe. Onde na maioria das vezes, essa mulher é vista apenas como responsável em gerar um filho. A sociedade impõe muito desse grupo de mulheres, e por consequência disso elas acabam demonstrando sentimentos de inferioridade e negatividade. Assim transformando essa mulher na única responsável por desempenhar a maternidade.

A importância da participação dos profissionais de saúde é fundamental e relevante para uma abordagem e acolhimento eficaz, utilizando de um olhar minucioso para perceberem as mulheres que procuram o serviço de saúde pela a necessidade referente à infertilidade, para isso eles precisam ser sensíveis, prestando uma atenção humanizada que assim possam promover uma rede de assistência digna para essas mulheres.

\section{Referências}

1. Júnior N.A, Reis, R.B.; Madeira, R.S. Manu: Manual de Urologia. São Paulo: PlanMark, 2010.

2. World Health Organization. Mother or nothing: the agony of infertility. Bull World Health Organ. 2010; 88:881-2, http://dx.doi.org/10. 2471/BLT. 10.011210

3. Instituto Brasileiro de Geografia e 
Estatística. Síntese de indicadores sociais. Estudos e pesquisas de informação demográfica e socioeconômica. 2005; 19:317.

4. Ministério da Saúde (Br). Portaria $\mathrm{n}$ 은 388, de 06 de julho de 2005. Brasília (DF): Ministério da Saúde; 2005.

5. Borlot Ana Maria Monteiro, Trindade, Zeidi Araújo. As tecnologias de reprodução assistida e as representações sociais de filho biológico. Estudos de psicologia, v. 9, n. 1, p. 6370, 2004.

6. Leite Renata Ramalho Queiroz, Frota, Ana Maria Monte Coelho. O desejo de ser mãe e a barreira da infertilidade: uma compreensão fenomenologica. Revista da Abordagem Gestáltica, v. 20, n. 2, p. 151-160, 2014.

7. Oliveira C.C. O luto pela criança que não nasceu. In: R. M. Melamed \& J. Quayle (Orgs.). Psicologia em reprodução assistida: experiências brasileiras. São Paulo: Casa do Psicólogo, p. 207220, 2006.

8. Lins Patrícia Gomes Accioly et al. O sentido da maternidade e da infertilidade: um discurso singular. Estud. psicol.(Campinas), v. 31, n. 3, p. 387-392, 2014.

9. Gradvohl Silvia, MayuMi Obana et al. Estresse de homens e mulheres que buscam tratamento para infertilidade. Revista Brasileira de Ginecologia e Obstetrícia, 2013.

10. Badinter E. L'amour en plus. Paris: Flammarion, 1980.

11. Scavone Lucila. Maternidade: transformações na família e nas relações de gênero. Interface-Comunicação, Saúde, Educação, v. 5, n. 8, 2001.

\section{Endereço para Correspondência}

Universidade do Estado do Rio Grande do Norte

e-mail: ellanygurgel@hotmail.com

Recebido em 14/02/2017

Aprovado em 14/07/2017

Publicado em 21/08/2017 